ASIAN JOURNAL OF PLANT
Website: http://journal.hibiscuspublisher.com/index.php/AJPB/index
PUBLISHER

\title{
Determination of the Detection Limit of the Detection of GMO in Food Using the Isothermal Solid-Phase Recombinase Polymerase Amplification on Microfluidic DVDs
}

\author{
Noor Azlina Masdor ${ }^{1 *}$ \\ ${ }^{1}$ Biotechnology Research Centre, Malaysian Agricultural Research and Developmental Institute (MARDI), \\ 43400 Serdang, Selangor, Malaysia. \\ *Corresponding author: \\ Dr. Noor Azlina Masdor, \\ Biotechnology Research Centre, \\ Malaysian Agricultural Research and Developmental Institute (MARDI), \\ 43400 Serdang, \\ Selangor, \\ Malaysia. \\ Email: azlina@mardi.gov.my
}

\section{HISTORY \\ Received: $15^{\text {th }}$ Oct 2021 \\ Received in revised form: $2^{\text {nd }}$ Dec 2021 Accepted: $18^{\text {th }}$ Dec 2021}

\section{KEYWORDS}

Limit of the Detection

GMO

Isothermal Solid-Phase Recombinase

Polymerase Amplification

Microfluidic DVDs

\begin{abstract}
A major drawback of the current literature on bioassay development is that these tests are not made using statistically robust methods for establishing the limit of detection. As an alternative, researchers often make use of simple detection-limit methods that are only roughly indicative of the actual detection limit. We can only assume that this is due to a practical need for simplified processes, in addition to the notion that the limit of detection theory has already been lowered to practice for bioassays. A DNA sensor based on light intensity of the scanning laser on a DVD drive with microfluidic layer etched onto the polycarbonate surface of an ordinary DVD has been previously developed for fast screening of genetically modified organisms (GMOs). The resultant calibration curve showed a sigmoidal calibration curve but was not modelled according to any of the sigmoidal models available. The objective of this study is the remodel the data using the standard 4-PL model and to determine the Limits of Detection (LOD) based on the standard method. The LOD value obtained through the 4PL modelling exercise based on a pooled standard deviation method yielded an LOD value of $62 \mathrm{mg} / \mathrm{g}$ (95\% confidence interval of 17 to 158 ), which was quite similar to the classical three standard deviation of the blank method but was lower than the rough estimation employed in the original publication.
\end{abstract}

\section{INTRODUCTION}

Biochemical diagnostic procedures, such as protein binding, rely on biomolecular interactions as its diagnostic modality, and as a consequence, their calibration curves are more complex. In addition, sigmoidal curves are often seen in these tests. In the event of asymmetry, a logistic (5PL) curve, or a logistic (4PL) curve, may be the best way to represent a distinctive sigmoidal relationship. It is possible that the linearization of an otherwise nonlinear connection by $\log$ transformation may result in a disruption of the error structure of the curve, and that this will have the opposite effect of decreasing or even eliminating error in the relationship.

The present literature on bioassay development does not make use of statistically robust techniques for determining the limit of detection of a particular test, which is a significant limitation. Instead, researchers often use basic techniques that give an approximate estimate of the detection limit, sometimes without any indication of confidence in the estimate. This absence of robust techniques is possibly owing to a practical desire for easy and simple procedures, as well as a lack of such approaches that have diminished the ideas of limit of detection theory to practise for bioassays.

Support materials, probes, assay formats and transduction events that are required to accomplish and monitor a biorecognition response are one of the most essential concerns in DNA biosensor research. It's because they can be made into devices that do everything from treating samples to managing fluids to performing detections all in one, plastic polymers have an advantage over silicon chips when used as analytical platforms [2-6]. The fluid management and signal detection systems are not easily suitable for a wide range of circumstances, even if the recommended platforms may be useful in real-world implementations in some cases. The creation of a semiautomated DNA test in microarray format is an enhanced format based on the integration of a simple adhesive microfluidic layer onto the polycarbonate surface of conventional DVDs. A spinning platform is used to store lyophilized chemicals, and primers are pre-printed on the DVD prior usage. Additionally, solid-phase amplification is used to adhere the amplified 
products to the DVD disc's surface after they are manually dispensed. Afterward, the microfluidic layers are separated, and the disc is scanned utilising a reflection mode of operation (i.e., conventional DVD drive). It is possible to create a microarray picture by utilising a data collection programme because of the existence of the amplification product, which changes the light intensity of the scanning laser on the DVD drive (in reflection mode). Proof-of-concept screening of genetically modified organisms has been carried out using this technology because it is low-cost, reliable, and quick [1]. The resultant curve showed a sigmoidal calibration curve but was not modelled according to any of the sigmoidal models available[1]. The objective of this study is the remodel the data using the standard 4-PL model and to determine the Limits of Detection (LOD) based on the standard method.

\section{Acquisition of Data}

Data from a published work [1] from figure 3 showing a calibration curve of GMO detection on the microfluidic disk from the GMO in a certified reference materials; maize Bt11 (ERM$\mathrm{BF} 412 \mathrm{~b}$ and ERM-BF412f). The data were processed using the software Webplotdigitizer 2.5 [7] which digitizes the scanned figure into a comma separated value [8].

\section{Four parameter logistics modelling}

In the assay, the calibration curves will be fitted with a non-linear regression using a four-parameter dose-response equation $[9,10]$ as following:

$$
y=\frac{a-d}{1+\left(\frac{x}{c}\right)^{b}}+d
$$

Where $\boldsymbol{y}$ signifies the response signal (optical density), $\boldsymbol{x}$ signifies the DNA $\log$ concentration, $\boldsymbol{a}$ and $\boldsymbol{d}$ signify the maximum and minimum signal response of the calibration curve, correspondingly, $\boldsymbol{b}$ is the Hill coefficient which represents the slope-like parameter and $c$ represents the DNA log concentration producing a $50 \%$ signal response $\left(\mathrm{EC}_{50}\right)$ value. Both the classical three times the standard deviation of the blank and another statistically robust technique for estimating the analytical LOD of a classic sigmoidal correlation based on the pooled standard deviation of datapoints will be utilized [11]. In the traditional LOD determination, a single test concentration which is usually taken from the lowest tested concentration or control will be utilized to obtain the standard deviation of the blank. Most assays are carried out using fewer than 10 replicates per concentration with the norm is three replicates per concentration level.

Anything less than 10 replicates per concentration have been shown to give a less accurate representation of the population variance and recommendation is to pool the standard deviation for all test samples [11]. When there are fewer than ten replicates per concentration level, the data points can be weighted by inverse variance to compensate for the variation in variance. Because this method is not recommended when there are less than 10 replicates per concentration level, an unweighted fit was used in this case instead.

Furthermore, it should be noted that the unweighted fit is consistent with the homoscedasticity criterion that was created for the initial pooled estimate of variance. The limit of detection (LOD) will then be calculated by averaging the mean value of absorbance at a blank concentration of DNA log concentration over three PSDs. The four-parameter logistics model and nonlinear regression analysis software will be used in tandem to calculate the LOD and execute regression analysis (PRISM, v 5.1) from www.graphpad.com.

\section{RESULT AND DISCUSSION}

ELISA-based and biological receptors-based or DNA-based standard curves are generally nonlinear and sigmoidal in property, and the best way to fit this kind of curve is to use a standard four-parameter logistic (4-PL) or the rarely used five parameter logistic (5-PL) models [12]. The raw data should then be fitted to the 4-PL curve through a modification of the curve model's parameters to achieve an ideal fitting between experimental and calculated data; the latter is often represented by a line running through the experimental data [13]. Although a patently sigmoidal profile was obtained by [1], the authors reported that the detection limits (LODs), calculated as the lowest amount of DNA that is able to produce a signal distinguished from the blanks were between 110 to $460 \mathrm{mg} / \mathrm{g}(0.011-0.046 \%)$.

The result in Fig. 1. shows a typical sigmoidal curve for the calibration curve based on the 4-PL equation. A typical sigmoidal profile was obtained. A good correlation coefficient value of 0.965 was obtained indicating good fitting.

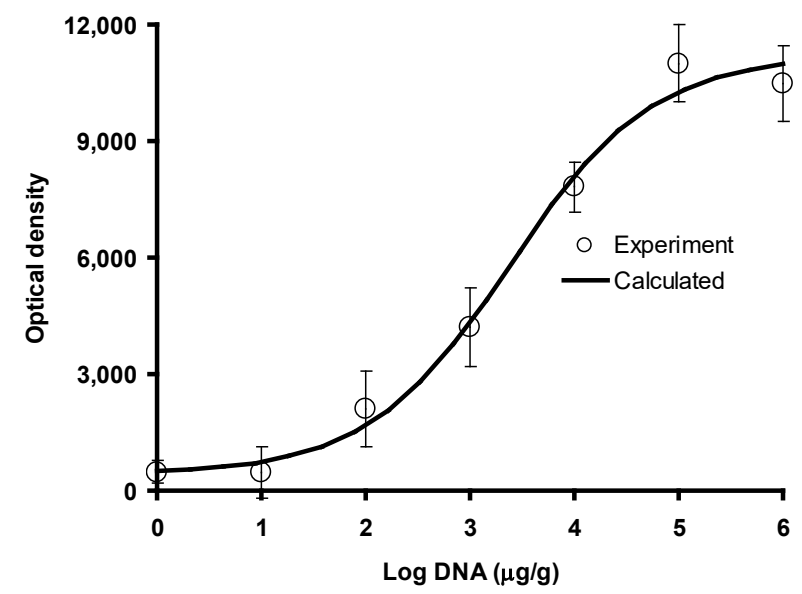

Isothermal solid-phase recombinase polymerase amplification on microfluidic digital versatile discs.

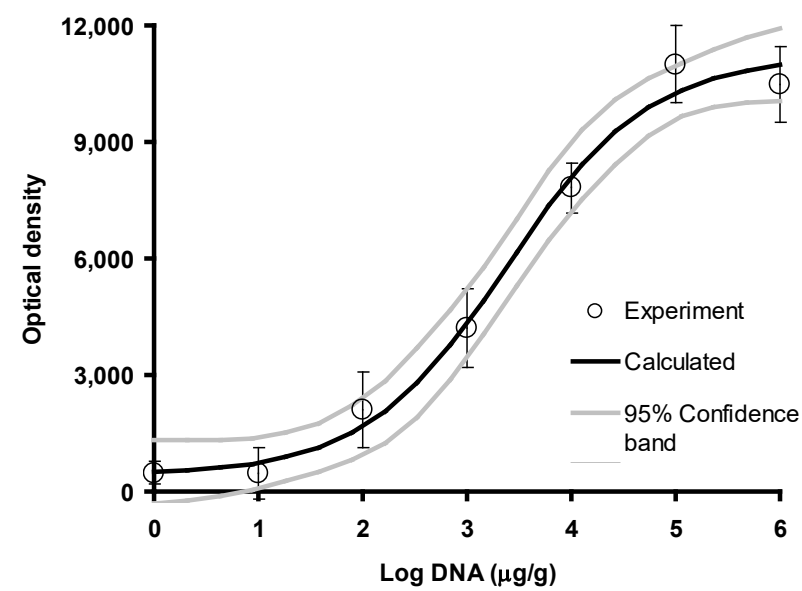

Isothermal solid-phase recombinase polymerase amplification on microfluidic digital versatile discs calibration curve, and its $95 \%$ confidence band. 
The value of the curve parameters is shown in the form of the four-parameter logistic equation as follows;

$y=418.0+\frac{10779}{1+10^{(3.384-x) * 0.636}}$

The LOD value obtained through the 4PL modelling exercise based on the classical method was $52 \quad \mathrm{mg} / \mathrm{g} \quad(95 \%$ confidence interval of 9 to 190) while the PSD method yielded an LOD value of $62 \mathrm{mg} / \mathrm{g}$ (95\% confidence interval of 17 to 158), which was quite similar to the classical method and was lower than the rough estimation employed by [1]. Because it is recommended that LOD values be computed using the 4-PL technique in the case that the curve has an obviously sigmoidal profile, the LOD value derived using the 4PL modelling approach should be utilised to report the LOD value in this situation.

Last but not least, in the event that the calibration curve has a sigmoidal profile, the 4PL model should be used to fit the data rather than a linear model, and the LOD value should be derived using the 4PL model rather than a linear model. According to the findings of this study, the adoption of the 4Pl model proved successful, as it was able to represent the entire date curve rather than just a linear section of it. When developing an ELISA technique, the linear part is crucial since it is a handy and speedy approach for determining the sensitivity of the method. It is also typically a more beneficial method in field applications when a quick and easy assessment is required. The 4Pl model, on the other hand, should not be abandoned because it is capable of reporting the LOD value and its 95 percent confidence range for any created technique with high accuracy.

\section{ACKNOWLEDGEMENT}

This work was supported by MARDI via WRM fund (TP-RB0023).

\section{REFERENCES}

1. Tortajada-Genaro L, Santiago Felipe S, Amasia M, Russom A, Maquieira A. Isothermal solid-phase recombinase polymerase amplification on microfluidic digital versatile discs (DVDs). RSC Adv. 2015 Mar 27;5.

2. Manzano M, Cecchini F, Fontanot M, Iacumin L, Comi G, Melpignano P. OLED-based DNA biochip for Campylobacter spp. detection in poultry meat samples. Biosens Bioelectron. 2015;66:271-6.

3. Gao Z, Tansil N. A DNA biosensor based on the electrocatalytic oxidation of amine by a threading intercalator. Anal Chim Acta. 2009;636(1):77-82.

4. Malic L a, Veres T c, Tabrizian M a b. Biochip functionalization using electrowetting-on-dielectric digital microfluidics for surface plasmon resonance imaging detection of DNA hybridization. Biosens Bioelectron. 2009;24(7):2218-24.

5. Wen S, Zeng T, Liu L, Zhao K, Zhao Y, Liu X, et al. Highly Sensitive and Selective DNA-Based Detection of Mercury(II) with $\alpha$-Hemolysin Nanopore. J Am Chem Soc. 2011 Nov 16;133(45):18312-7.

6. Che-Engku-Chik CEN, Yusof NA, Abdullah J, Othman SS, Said MHM, Wasoh H. Detection of tuberculosis (TB) using gold standard method, direct sputum smears microscopy, PCR, qPCR and electrochemical DNA sensor: A mini review. J Biochem Microbiol Biotechnol. 2016 Dec 30;4(2):16-21.

7. Rohatgi$$
\text { A. }
$$

WebPlotDigitizer. http://arohatgi.info/WebPlotDigitizer/app/ Accessed June 2 2014.; 2015.

8. Khare KS, Phelan Jr FR. Quantitative comparison of atomistic simulations with experiment for a cross-linked epoxy: A specific volume-cooling rate analysis. Macromolecules. 2018;51(2):56475.

9. Iturria SJ. Statistical inference for relative potency in bivariate dose-response assays with correlated responses. J Biopharm Stat. 2005;15(2):343-51.

10. Masdor NA, Altintas Z, Shukor MY, Tothill IE. Subtractive inhibition assay for the detection of Campylobacter jejuni in chicken samples using surface plasmon resonance. Sci Rep. 2019 Sep 20;9(1):13642.

11. Holstein CA, Griffin M, Hong J, Sampson PD. Statistical method for determining and comparing limits of detection of bioassays. Anal Chem. 2015 Oct 6;87(19):9795-801.

12. Masdor NA, Altintas Z, Tothill IE. Sensitive detection of Campylobacter jejuni using nanoparticles enhanced QCM sensor. Biosens Bioelectron. 2016;78:328-36.

13. Cumberland WN, Fong Y, Yu X, Defawe O, Frahm N, De R. Nonlinear Calibration Model Choice between the Four and FiveParameter Logistic Models. J Biopharm Stat. 2015;25(5):972-83. 